\title{
Chaos and Bifurcation of Control Feedback System Using Variational Iteration Method
}

\author{
Evuiroro Edirin Judith, Ojarikre Henritta Ify \\ Department of Mathematics, Delta State University, Abraka, Nigeria \\ Email address: \\ ojarikreify@delsu.edu.ng (E. E. Judith), judy4edirin@yahoo.com (O. H. Ify)
}

\section{To cite this article:}

Evuiroro Edirin Judith, Ojarikre Henritta Ify. Chaos and Bifurcation of Control Feedback System Using Variational Iteration Method. Applied and Computational Mathematics. Vol. 10, No. 4, 2021, pp. 86-90. doi: 10.11648/j.acm.20211004.11

Received: May 19, 2021; Accepted: June 15, 2021; Published: July 9, 2021

\begin{abstract}
Chaos theory discusses the behavior of some complex systems which are sensitive to initial condition. It entails some interesting properties such as space-filling, sensitivity to initial conditions, control synchronization and dynamics which can be accessed using different control methods. This paper considers a non-linear feedback control system of underlying symmetries of chaos and bifurcation with periodic equations. Bifurcation theory plays a very vital role in the analysis of Chaos dynamics. Therefore, bifurcation process of chaotic systems involving Lyapunov exponents are studied. The work describes nonlinear systems where small changes produce notable change in the space phase with all the possible states corresponding to a unique point. A Variational Iteration Method (VIM) is adopted to determine the solution stability and bifurcation paths of the dynamic system. Thus. in the paper, state problems of the system are decomposed using Lagrange multiplier to obtain the Adomian polynomials. The polynomials generated in turn minimize the problem by providing an approximate solution that is very close to the analytic solution. A numerical illustration has been presented of a nonlinear coupled equation using VIM. Illustrations showing the graph of the phase space structure, the paths displayed in motion and the region of stability of the numerical scheme was obtained. The result shows that the chaotic nonlinear system is stable.
\end{abstract}

Keywords: Chebyshev Polynomial, Lagrange Multiplier, Variational Iterative Method, Chaos and Bifurcation

\section{Introduction}

Radzicki [17] and Butler [4] have amongst others observed that chaotic systems that tend to be characterized by nonlinear relationships transform dynamically. That is, complex interactions are recreated over time. This discoveryhas led to an increasing interest in the applicationofchaos theory to a height of recorded fields like ecology, medicine, international relations and economics. Chaos exhibited some interesting properties like initial conditions sensitivity, synchronization and dynamics which can easily be accessed using various control methods. Mammeri [12] studied these properties using numerical investigation. A sinusoid map in 3-D discrete map which produces chaotic attractors was observed to be periodic. In addition, there are chaotic systemsthat have periodic orbits that survives in the boundaries of strong perturbation with integral evaluation. The boundary between chaotic and regular behaviours is usually characterized according to Strogatz [18] by progressive periodic doubling, which is followed byperiodquadrupling. But a more definitive chaotic behavior was experienced using lyapunov exponent. A good example of this periodic display is time series delay plots. This series graph provides the best argument for the claims made in [12] and shows a clear transition from periodicity to irregularity. On analyzing a special chaotic model, Briggs and Peat [3] suggested that, an interpretation of chaos with respect to the relationship between the job market and employees for instance, could be made by using the model of career counseling. This suggests that any random situation can be interpreted in terms of Chaos theory. Differential equations werealsoinitiated in the Chaos analysis as it was in diverse disciplines (Lorenz, [10]). Gleick [5] and Hayles [6] was convinced that Chaos was discoveredby Lorenz and acknowledged him as one of the first contributors to recognizechaos theory. Consequent upon that, with three sets of small equations, they realized that there was only one explanation for theturbulent behaviour and fratal results 
when plotted as a phase space. For some dynamic systems, phase space diagrams are straightforward but in Lorenz's system, the space diagram emerged like a pair of wings of butterfly (Lorenz, [9]).

Chaos is also a mathematical tool which could reveal not just patterns within but also show similar patterns betweenranges of unrelated physical processes (Kellert [7]). At the front burner of discoveries was the work of Mandelbrot [13], who had carried out immense work in the fractal geometry and his findings has revealed outstanding images. These images are similar to the same ones investigated by Lorenz equations in Lorenz [10]. [9]'s significant contribution was to create iterative solution technique and show progressively the resulting space diagrams of the modeled equation by varying the initial conditions. There are two types of orders in chaos, 'order within chaos' and order out of chaos'. When those in support of 'order within chaos' were pursuing the existence of this fact, the other branches of chaos theory was emerging due to the discoveries in the work of Levy [8]. The focus in [8] was on different strategies which exhibit the emergence of order from disorder and the starting of chaos theory in general. Time is irreversible (Porush [15]) The essential change is to see chaos as an action that which makes order possible. Chaos is the womb of life, not its tomb (Hayles [6]). Pyragas [16] analyzed the remarkable behavior of dissipative structures from the space diagrams, and noted the processes of continuous control of the observed chaotic behaviour of the system areself controlling feedback. [16] concluded thatthe problem of the stability of a systemand how to stabilize the unstable periodic features of a chaotic system could be achieved without force.. This kind of chaos controlhaving time continuous perturbation may be formulated as combined feedback or self controlling feedback as in Ott et al [14].

Hence, this current study takes a look at the random behavior of these systems and the pattern resulting from such display. Although chaos theory have been described in terms ofthe twoapproaches [6]. These approachesfocusedmore on order within chaos, and the dissipative structure (PHYSICAL transformation) shifting attention to order out of chaos. The statement thatchaotic systems aredetermined by initial conditions is a fundamental feature of the two aspectsMENTIONED ABOVE. nOTABLEcharacteristics IS

$$
u_{i+1}(x)=u_{i}(x)+\int_{0}^{x} \lambda(s)\left(L u_{i}\right.
$$

$\lambda(s)$ is a general Lagrange's multiplier, observe that for this method $\lambda$ could be a constant or a function, and $\tilde{u}_{n}$ is a restricted value (behaving as a constant), therefore $\delta \tilde{u}_{n}=0$, where $\delta$ is avariational derivative. Also, the Lagrange multiplier $\lambda(s)$ (see [1]) is determined using the formula

$$
\lambda(s)=(-1)^{n} \frac{(s-x)^{(n-1)}}{(n-1) !},
$$

where $\mathrm{n}$ in (1) is the order of the highest derivative. Algorithms of Variational Iteration Method
THE nonlinearityPROPERTY, whereby large effects can be brought about by Global theories WHICHdefine the behavior for allPOINTSlocatED within the system. Only IN recent RESEARCHES, local theories have been developed-theories which define behaviorAL rules for isolated local regions within the larger systemUNDER STUDY. The spontaneous appearance of Islands of order from a sea of chaos explainS why local theories are essential. Many researchers formulated their theories to explain the phenomenaoflocalorglobal universally. The distinction between recent discovery and the classical belief is a scale invariant.

Chaos theory, in contrast, shows that scalingmethod is generally vital for complexdynamic systems. A comment in [6] on this issueof scaling provides a final word on the issue of localization versus globalization. The science of chaos celebrates the disorder that earlier works ignored, that is in seeing chaos, not as an obstacle to scientific progress but more as information that rescues the world from repetition. If the focus is on the structures defining chaos with its underlying reoccurring symmetries, then the structures causing chaos can be revealed and analytical solutions achieved.

\section{Variational Iteration Method (VIM)}

VIM established by Ji-Huan (Abbasbandy and Shivanian [1]) is now used to handle a wide variety of linear and nonlinearequatios, homogeneous and inhomogeneous systems. The method producesafast convergent and successive approximations of the exact solution which are not component entities if the solution exists. This computational approach also handles both nonlinear and linear problems in the same way with or without requiring any specific restrictions. Moreover, the method obtains the solution in a series form whichconverges if the exact solution exists. The resulting series can then be employed for numerical purposes when the exact solution is not obtainable. We now present the steps for the method.

Consider the general differential equation of the form:

$$
L u(x)+N u(x)-g(x)=0
$$

Now, the functional for Equation (1) can be constructed as follow:

$$
(s)+\widetilde{N} u(s)-g(s)) d s, i \geq 0
$$

We employ the standard VIM as seen in Mamadu and Njoseh [11] and also Anjum and HE [2]. Given the nonlinear equation of $K t h$ order as

$$
u^{(k)}(t)+f\left(u, u^{\prime}, u^{\prime \prime}, \ldots, u^{(k)}\right)=0
$$

Then VIM formulation can be constructed as follows:

$$
u_{n+1}(t)=u_{n}(t)+\int_{0}^{1} \lambda\left(u_{n}^{(k)}+\widetilde{f}_{n}\right) d s,
$$

where $\partial \widetilde{f_{n}}=0, f_{n}=f\left(u, u_{n}^{\prime}, u_{n}^{\prime \prime}, \ldots\right)$. Introducing the 
multiplier, we have

$u_{n+1}(t)=u_{n}(t)+\int_{0}^{1} \frac{1}{(n-1) !}(s-t)^{n-1}\left[u_{n}^{(k)}(s)+f_{n}\right] d s$,

The major advantage of this iteration formula is that the initial solution $u_{0}(t)$ can be freely chosenwith an unknown parameters in the formulationand the disadvantage is that some repeated and unnecessary iterations are involved in the algorithm at each step of the iteration.

After identifying the Lagrange multiplier $\lambda$ in (3), the condition of initial approximation $u_{0}$ must satisfy the given initial/boundary conditions. This is the major shortcoming of this method.

$$
u_{n+1}=-L^{-1}\left(R\left(u_{n}\right)\right)-L^{-1}\left(A_{n}\left(u_{0}, u_{1}, \ldots, u_{n}\right)\right),
$$

or equivalently,

$$
\begin{gathered}
u_{0}=L^{-1} g, \\
u_{1}=-L^{-1}\left(R\left(u_{0}\right)\right)-L^{-1}\left(A_{0}\left(u_{0}\right)\right), \\
u_{2}=-L^{-1}\left(R\left(u_{1}\right)\right)-L^{-1}\left(A\left(u_{0}, u_{1}\right)\right),
\end{gathered}
$$

Consider the nonlinear function $f(u)$. Then, the infinite series generated by applying the Taylor's series expansion of $f$ about the initial function $u *_{0}$ is given by

$$
f(u)=f\left(u_{0}\right)+f^{\prime}\left(u_{0}\right)\left(u-u_{0}\right)+\frac{1}{2 !} f^{\prime \prime}\left(u_{0}\right)\left(u-u_{0}\right)^{2}+\cdots
$$

By substituting (7) into equation (8), we have:

$$
f(u)=f\left(u_{0}\right)+f^{1}\left(u_{0}\right)\left(u_{1}+u_{2}+\cdots\right)+\frac{1}{2 !} f^{\prime \prime}\left(u_{0}\right)\left(u_{1}+u_{2}+\cdots\right)^{2}
$$

Now, to expand (9), one needs to determine the order of each term in (9) which actually depends on both the subscripts and the exponents of the $u_{n}{ }^{\prime} s$.

As a result, rearranging the terms in the expansion (9) according to the order, we have

$$
\begin{gathered}
f(u)=f\left(u_{0}\right)+f^{\prime}\left(u_{0}\right) u_{1}+f^{\prime}\left(u_{0}\right) u_{2}+\frac{1}{2 !} f^{\prime \prime}\left(u_{0}\right) u_{1}^{2}+f^{\prime}\left(u_{0}\right) u_{3}+\frac{2}{2 !} f^{\prime \prime}\left(u_{0}\right) u_{1} u_{2}+\frac{1}{3 !} f^{\prime \prime \prime}\left(u_{0}\right) u_{1}^{3}+f^{\prime}\left(u_{0}\right) u_{4}+ \\
\frac{1}{2 !} f^{\prime \prime}\left(u_{0}\right) u_{2}^{2}+\frac{2}{2 !} f^{\prime \prime}\left(u_{0}\right) u_{1} u_{3}+\frac{3}{3 !} f^{\prime \prime \prime}\left(u_{0}\right) u_{1}^{2} u_{2}+\frac{1}{4 !} f^{\prime \prime \prime \prime}\left(u_{0}\right) u_{1}^{4}+\cdots
\end{gathered}
$$

Considering the nonlinear function $f(u)$, the infinite series generated by applying the Taylor's series expansion of $f$ about the initial function $u_{0}$ is given by

$$
\begin{gathered}
f(u)=f\left(u_{0}\right)+f^{\prime}\left(u_{0}\right) u_{1}+f^{\prime}\left(u_{0}\right) u_{2}+\frac{1}{2 !} f^{\prime \prime}\left(u_{0}\right) u_{1}^{2}+f^{\prime}\left(u_{0}\right) u_{3}+\frac{2}{2 !} f^{\prime \prime}\left(u_{0}\right) u_{1} u_{2}+\frac{1}{3 !} f^{\prime \prime \prime}\left(u_{0}\right) u_{1}^{3}+f^{\prime}\left(u_{0}\right) u_{4}+ \\
\frac{1}{2 !} f^{\prime \prime}\left(u_{0}\right) u_{2}^{2}+\frac{2}{2 !} f^{\prime \prime}\left(u_{0}\right) u_{1} u_{3}+\frac{3}{3 !} f^{\prime \prime \prime}\left(u_{0}\right) u_{1}^{2} u_{2}+\frac{1}{4 !} f^{\prime \prime \prime \prime}\left(u_{0}\right) u_{1}^{4}+\cdots
\end{gathered}
$$

\section{Local Nonlinear Stability Associated with Feedback Linearization}

We find a set of simple coordinate transformationsthattries to convert the original nonlinear time-periodic control problem to anequivalent dynamic linear system with periodT. supposea linear control equation is designed from the existing linear methods. The essence of the linear dynamic feedback control is to stabilize the nonlinear system since it provides an increased region of stability. We shall consider the nonlinear periodic system

$$
\begin{gathered}
\dot{x}=F(x, u(x), \varphi, t)+g(x(t) u) \\
y(t)=\mathrm{h}(u(t))
\end{gathered}
$$

$x(t)$ is a state vector in an open $\operatorname{subset} D \in \mathcal{R}^{n}, u(t) \in$ $\mathcal{R}^{m}$ is the control vector while $y(t) \in \mathcal{R}^{m}$ y (t) is the output vector $f: D \rightarrow \mathcal{R}^{n}, \mathrm{~h}: D \rightarrow \mathcal{R}^{m}$ and $g: D \rightarrow \mathcal{R}^{n x m}$ are smooth

$$
\frac{\mathrm{d}}{\mathrm{dt}} \mathrm{x}_{1}(\mathrm{t})=\mathrm{x}_{2}(\mathrm{t})^{2}-4, \frac{\mathrm{d}}{\mathrm{dt}} \mathrm{x}_{2}(\mathrm{t})=\mathrm{x}_{1}(\mathrm{t})-1+\mathrm{u}(\mathrm{t}), \mathrm{x}(\mathrm{t})=\mathrm{x}_{1}(\mathrm{t})+\mathrm{x}_{2}(\mathrm{t}),
$$

with initial conditions with initial conditions functions. That is $\mathrm{f}, \mathrm{h}$ and $\mathrm{g}$ are continuously differentiable. Suppose the system is governed by the evolution equation,

$$
u(x)=\alpha_{i}(x)+\beta(x) v, \beta(x)=\gamma(0)
$$

Then (11) yields

$$
\dot{x}=A x+B x
$$

alinear system having parameters $\alpha_{i} \in\left(\alpha_{j}, \alpha_{h}\right)$ that are fixed. The transformation equation of (12), are given by equations (13-15) which arethefeedback linearization. Usually, the major problem in the analysis of (12) startswith a changes in the control variable, (15) becomes realistic. The process depends more on the underlying information available about the system (12).

\section{Numerical Examples}

Consider the nonlinear coupled system 


$$
\mathrm{u}(\mathrm{t})=0, \mathrm{x}_{1}(\mathrm{t})=2, \mathrm{x}_{2}(\mathrm{t})=4 \text { and constraints }=\left[0<\mathrm{x}_{1}(\mathrm{t})<\mathrm{x}_{2}(\mathrm{t})\right]
$$

Therefore, using VIM, we obtain the following iterative formulas:

$$
\begin{gathered}
x_{1_{n+1}}(t)=x_{1_{n}}(t)-\int_{0}^{t}\left(\frac{d}{d t} x_{1_{n+1}}(s)-x_{1_{n+1}}^{2}(s)+4\right) d s, n \geq 0, \\
x_{2_{n+1}}(t)=x_{2_{n}}(t)-\int_{0}^{t}\left(\frac{d}{d t} x_{2_{n+1}}(s)-x_{1_{n+1}}{ }^{2}(s)+1+u(s)\right) d s, n \geq 0,
\end{gathered}
$$

satisfying the conditions above.

Our aim is to seek the region of stability and bifurcation and not just obtaining approximations to guarantee convergence of the chaotic theory as required. Hence, implementing the iterative schemes (17) and (18) for $n \geq 0$, subject to the initial constraint, we obtain:

$$
\begin{gathered}
{\left[\mathrm{x}_{1}(\mathrm{t})=1.49999999768708, \mathrm{x}_{2}(\mathrm{t})=-2.00000001053627\right],} \\
{\left[\frac{\mathrm{d}}{\mathrm{dt}} \mathrm{x}_{1}(\mathrm{t})=4.21450963017378 \times 10^{-8}, \frac{\mathrm{d}}{\mathrm{dt}} \mathrm{x}_{2}(\mathrm{t})=-4.6258464128 \times 10^{-9}\right],} \\
{[u(t)=-0500000002312923],[y(t)=-0.500000012849197]}
\end{gathered}
$$

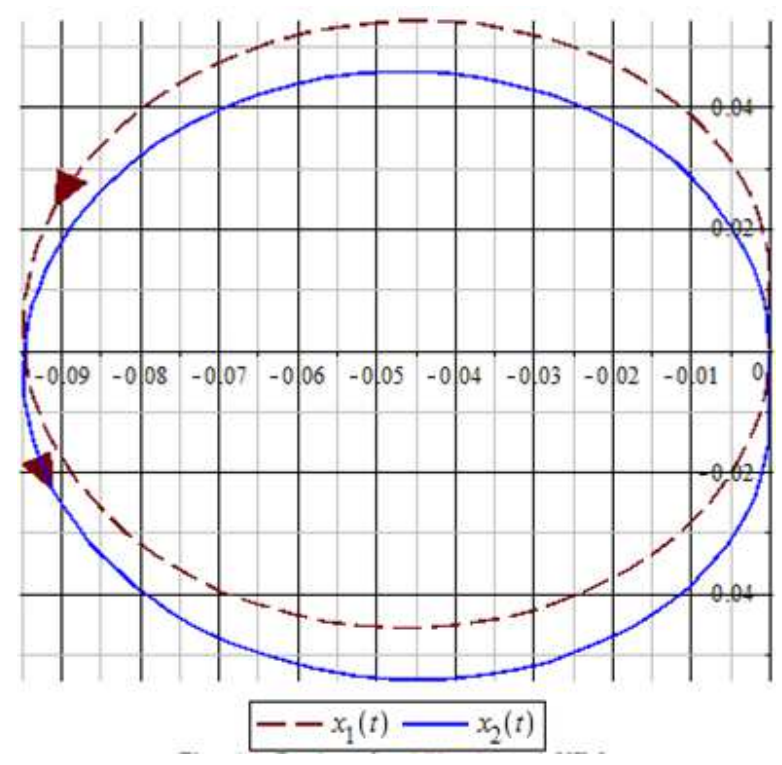

Figure 1. Region of stability through VIM.

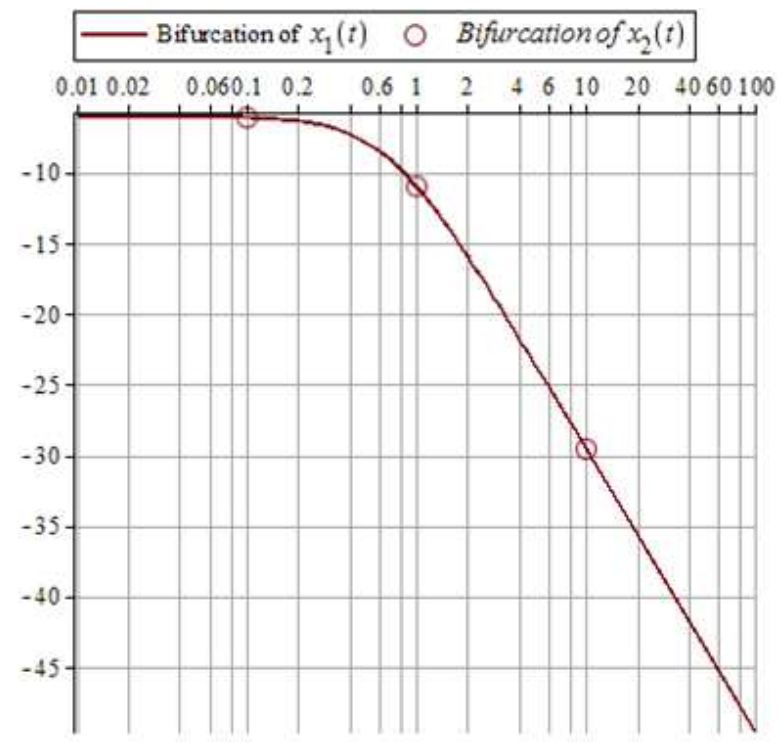

Figure 2. Bifurcation of the nonlinear coupled dynamical system.
The example above is illustrated graphically in figures 1 and 2 by obtaining the region of stability and bifurcation of the nonlinear coupled dynamical system (18) through VIM.

Using VIM for the coupled dynamical system together with the constraints and initial condition, it was observed that the system is asymptotically stable based on the illustration above. The feedback acts by changing the parameter. Also, the chaotic behaviour of the system in terms of its stability and bifurcation has been shown in the figures 1 and 2. This results show that the dynamic system though being coupled exhibits some sense of stability within the region of 0 to 0.095 . This was first linearized by decomposition with the aid of Adomian polynomials,

For further evaluation in VIM, especially for the nonlinear term in equations (1) or (2) these can be decomposed using

$$
N u(x)=\sum_{n=0}^{\infty} A_{n}(x, t)
$$

The $A_{n}$ in (19) is the Adomian polynomial. This show that the ADM is preferable for solving nonlinear systems.

\section{Conclusion}

The science of chaos shares and celebrates the disorder that earlier scientists ignored which now reduces sterile repetition. On the other hand, the study of chaos within the system shows that when one focuses on the underlying recursive symmetries, accurate system equations and space diagram can be obtained revealing that analytical solutions can be achieved. This has been illustrated using VIM.

\section{References}

[1] Abbasbandy, S and Shivanian, E. Application of VIM for System of nonlinearVolterra'sIntegro-Differential Equations. Mathematical and ComputationalApplications. 14 (2), 147158, 2009. 
[2] Anjum, N. and He, J. H. Laplace Transform: Making Variational Iteration Method Easier. Elsevier-Journal of Applied Mathematics Letters, 16 (1): 134-138, 2019 http://doi.org/10.1016/j.aml.2019.01.016.

[3] Briggs, J. \& Peat, F. D. (2000) Seven Life Lesson of Chaos: Spiritual Wisdom from the Scienceof Change. New York.

[4] Butler, A. A Methodological Approach to Chaos. Federal Reserve Bank of St. Louis 72 (13), 36-48. 1990.

[5] Gleick, J. Chaos: Making a New Science. New York: Viking Penguin, 1987.

[6] Hayles, N. K. Chaos Bound: Orderly Disorder in Contemporary Literature and Science. Ithaca: Cornell University Press, 1990.

[7] Kellert, S. H. In The Wake of Chaos Unpredictable Order in Dynamical Systems. Chicago: University of Chicago Press, 1993.

[8] Levy, D. L. Chaos Theory and Strategy: Theory. Application and Managerial Implications. Strategic Management Journal, 15: 167-178, 1994.

[9] Lorenz, E. N, Deterministic non periodic flow. Journal On Atmospheric Science, 20, 130-141, 1963.

[10] Lorenz, E. 'Predictability: Does the flap of a butterfly swings in Brazil set off a tornado in Texas?' Paper presented at the meeting of the American Association for the Advancement of Science, Washington DC, 1972.
[11] Mamadu, E. J and Njoseh, I. N. Variational Iteration Method for the Approximate Solution of Nonlinear Burger's Equation, Nigerian Journal of Basic and Applied Sciences, 24 (1), 7075, 2016.

[12] Mammeri, M. Symmetry and Periodic-Chaos in 3-DSinusoid Discrete Map, Bulletin of Mathematical Analysis and Applications, 9 (1), 1-8, 2017.

[13] Mendelbrot, B. Fractals, Chance and Dimensions. San Francisco: W. H. Freeman \& CO, 1977.

[14] Ott. E, Grebogi. C, Yorke. J. A (1990), Controlling chaos. Physical Review Letters 64: 1196-1199.

[15] Porush, D. Fictions as Dissipative Structures. Prigogines Theory and Postmodernism Road show in Chaos and Order. Complex Dynamics in Literature and Science. Chicago: The University of Chicago Press, 1991.

[16] Pyragas K (1992), Continuous control of chaos, by selfcontrolling feedback, Physics Letters 170: 421-428, 1992.

[17] Radzicki, M. J. Institutional Dynamics, Deterministic Chaos and Self Organizing System. Journal of Economic Issues, 24 (1) $57-102.1990$.

[18] Strogatz, S. H, Nonlinear Dynamics and Chaos. AddisonWeseley Publishing Company. United State Of America. 1994. 